\title{
Childhood Epileptic Encephalopathy in Sokoto, Northwestern Nigeria
}

\author{
MM Ahmad* and H Ahmed \\ Department of Pediatrics, Usmanu Danfodiyo University Teaching Hospital, Sokoto, Nigeria
}

\begin{abstract}
Background: Pediatric epileptic encephalopathies comprises a group of severe childhood epilepsy syndromes characterized by refractory seizures associated with progressive cerebral dysfunction, manifesting as cognitive or learning difficulties and behavioural or sensory impairments.
\end{abstract}

Objectives: To describe the clinical and electroencephagraphic profile of children seen with epileptic encephalopathy in Sokoto, Northwestern Nigeria.

Methodology: This is a descriptive report of cases of epileptic encephalopathy seen in a Pediatric Neurology Clinic, over a 5-year period (Jan. 2015 to Dec. 2020).

Results: Twenty-one cases that satisfied the definition criteria for childhood epileptic encephalopathy where identified over the study period, out of a total number 413 children diagnosed with epilepsy over the period, giving a hospital prevalence of $5.1 \%$. Three of the cases were excluded due to incomplete data.

Four (4) different syndromes of EE were recorded, namely: Epileptic spasms/West syndrome, Lennox-Gastaut syndrome, Doose syndrome and Landau-Kleffner syndrome. Epileptic spasm was the most prevalent type recorded in 7 (38.9\%) cases, followed by Lennox-Gastaut syndrome in 6 (33.3\%), Landau-Kleffner syndrome in $3(16.7 \%)$ and Doose Syndrome in $2(11.1 \%)$ in that order.

The mean age at diagnosis was $17.00 \pm 6.50$ months, with a male to female ratio of 1.6:1. There was a significant association between age of seizure onset and the type of seizure syndrome.

Conclusion: Pediatric epileptic encephalopathies are prevalent in our society, with epileptic spasms/West syndrome and Lennox-Gastaut syndromes leading the list. Perinatal asphyxia was the main risk factor identified among the cases.

\section{Keywords}

Encephalopathy, Epilepsy, Infantile spasms, Lennox-Gastaut, Doose syndrome, Landau-Kleffner syndrome

\section{Introduction}

Encephalopathy is a descriptive term referring to diffuse brain disorder leading to altered brain function and/or structure. Encephalopathy is not a single disease but a group of disorders with multiple causes, including metabolic, infective, traumatic, ischaemic or toxic causes.

The term epileptic encephalopathy (EE) was introduced by the International League Against Epilepsy (ILAE) 2001 diagnostic scheme, as a condition in which epileptiform abnormalities are believed to contribute to the severe cognitive and behavioural impairments beyond what is expected from the underlying pathology [1,2]. Although childhood EEs are individually rare, they collectively exert a significant burden on the child, the family and the healthcare system [3].

An incidence of $0.44 / 1000$ per year was reported in
Australia in 2019 [4]. However, there is dearth of information on childhood EE among African children with epilepsy. Therefore this study is aimed at reporting the electro-clinical presentations of these cases in Sokoto, Northwestern Nigeria. To the best of our knowledge, this is the first specific report on the profile of childhood EE among Nigerian children as well as West African sub-region.

*Corresponding author: MM Ahmad, Department of Pediatrics, Usmanu Danfodiyo University Teaching Hospital, Sokoto, Nigeria

Accepted: September 07, 2021

Published online: September 09, 2021

Citation: Ahmad and Ahmed (2021) Childhood Epileptic Encephalopathy in Sokoto, Northwestern Nigeria. J Pediatr Neurol Neurosci 5(2):164-168 
Generally, up to eight (8) age-related syndromes of childhood EE are recognized [5]. They can manifest during neonatal, infancy, early or late childhood, depending on the type and its possible aetiology. They all share some common features such as severe and frequent seizures, developmental problems, diffuse background inter-ictal epileptiform abnormalities and poor treatment outcomes. The continuous epileptic/epileptiform activity is believed to contributes to progressive cerebral dysfunction $[3,6,7]$. However, optimal treatment of the seizures may improve the cognitive and behavioural functions [3].

The underlying causes of childhood EE are diverse, with many of them attributable to static structural brain lesions such as cortical dysplasias, neurodegenerative, metabolic, genetic or chromosomal disorders $[4,6,8]$. Stödberg, et al. [9] and Ware, et al. [4] have reported up to $65 \%$ and $81 \%$ identifiable aetiologies respectively, in cohorts of children presenting with epilepsy syndromes in the first 2-years of life in Stockholm and Tasmania, mainly from structural and genetic aetiologies.

The current list of the childhood of the childhood EE include: Dravet syndrome (severe myoclonic epilepsy of infancy), Ohtahara syndrome (Early infantile epileptic encephalopathy), Infantile/epileptic spasms, Doose syndrome, Lennox-Gastaut syndrome, Landau-Kleffner syndrome, Continuous spike waves of slow sleep (CSWSS), and severe epilepsy with multiple independent spike foci $[3,5,6,10]$.

Many cases are clinically sporadic, but a comprehensive list of genes have been identified to be involved in the aetiology of childhood EE [4,10-13], as a result of the use of molecular genetic studies. Implicated genes play vital roles in brain synapse formation, action potential and neurotransmitter release [11]. De novo mutations involving genes encoding neuronal proteins have also been demonstrated in sporadic cases [8], as well as involvement of more than one gene in some EE such as Ohtahara (KCNQ2 and SCN2A genes) and Dravet (SCN1A, SCN2A SCN1B genes) syndromes [13]. Identification of the aetiology is important particularly for prognosis and genetic counseling [4,12], as well as halting the need for unnecessary investigations.

Inter-ictal EEG tracings in EE are always abnormal, revealing the encephalopathic nature of these syndromes as against other non-encephalopathic epilepsies that frequently revealed normal inter-ictal EEG background features. Common areas of EEG abnormalities are in the background activity, presence of epileptiform abnormalities and other nonspecific EEG findings.

\section{Patients and Methods}

This is a descriptive report of cases of epileptic encephalopathy seen in the Pediatric Neurology Clinic of Usmanu Danfodiyo University Teaching Hospital Sokoto, Northwestern Nigeria, over a 5-year period (Jan. 2015 to Dec. 2020). The Clinic serves as a major referral centre for Sokoto state and the neighbouring Nigerian states of Kebbi, Niger and Zamfara as well as neighbouring communities in Niger
Republic. Neonatal seizures and other cases with incomplete data were excluded.

A study proforma was used to record patients' relevant demographic and clinical presentations about the seizures. These included age, gender, age at onset of the seizures, age at diagnosis, the seizure type, presence of comorbidities, duration of treatment and response to treatment. Interictal EEG findings were also recorded for each patient. EEG's were taken in awake and/or in sleep states, depending on the child's state and ability to remain calm and cooperative. The duration of the EEG recording was 30-45 minutes for each patient. EEG findings were analyzed for each patient, including background activity, reactivity, description of the epileptiform features and final impression about the EEG.

Data was analyzed using SPSS version 23 and results were presented as simple proportions as well as means and standard deviations. A $P$-value less than 0.05 was considered statistically significant. Ethical clearance was obtained from the Hospital ethics committee.

\section{Results}

Twenty-one (21) cases of childhood EE were identified during the study period, out of 413 cases of childhood epilepsy seen during the period, giving a hospital prevalence of EE of $5.1 \%$ of all the cases of epilepsy. Three (3) cases had incomplete data record and were excluded, leaving 18 cases that were finally analysed.

Identified cases comprised of four (4) syndromes, namely: Infantile/epileptic spasms, Lennox-Gastaut syndrome (LGS), Doose syndrome (myoclonic-astatic epilepsy) and LandauKleffner syndrome (LKS). Infantile/epileptic spasms was the most prevalent syndrome recorded, in 7 of the 18 cases (38.9\%), followed by LGS in 6 (33.3\%) cases, LKS in3 (16.7\%) cases and Doose syndrome in $2(11.1 \%)$ cases, in that order.

The mean age of the patients was $5.37 \pm 4.08$-years. Eleven (11) of the 18 cases (61.1\%) were males, giving a male to female ratio of $1.6: 1$. The mean age at seizure onset was $9.6 \pm 1.45$ months, with $72.2 \%$ of the cases having their seizure onset during infancy period $(p=0.015)$, with a mean age at diagnosis (and treatment) of $17.00 \pm 1.65$ months.

A significant association was demonstrated between the age of seizure onset and the type of seizure $(p=0.015)$. There was no significant relationship between gender and age of seizure onset $(p=0.295)$, nor that of gender and seizure type ( $p=0.500)$.

Cerebral palsy and speech delays were the most frequent co-morbidities identified among the study cases. All the studied cases had abnormal EEG patterns demonstrable in the background activity as well as epileptiform abnormalities (mostly generalized and multifocal epileptiform discharges). Hysparrythmias were also demonstrated in three (3) of seven (7) cases with epileptic spasms/West syndrome. Brain imaging (CT/MRI) showed cerebral atrophy in majority of cases, with a case each (5.6\%) showing non-active hydrocephalus, sub cortical infarctions and periventricular calcifications, presumed to be from adverse perinatal events. 
Table 1: Distribution of cases based on gender and age at onset of seizures.

\begin{tabular}{|l|l|l|l|l|}
\hline \multirow{2}{*}{ Age at seizure onset } & \multicolumn{2}{|c|}{ Gender } & Total & Percentage \\
\cline { 2 - 5 } & Male & 8 & Female & \\
\hline$<12$ months & 8 & 5 & 13 & 72.2 \\
\hline $12-60$ months & 3 & 2 & 5 & 27.8 \\
\hline$>60$ months & 0 & 0 & 0 & 0 \\
\hline Total (\%) & $11(61.1)$ & $07(38.9)$ & 18 & 100 \\
\hline
\end{tabular}

Table 2: Type of EE and gender distribution among the cases.

\begin{tabular}{|l|l|l|l|l|}
\hline Seizure type & ES/IS & LGS & LKS & DS \\
\hline Males & 4 & 3 & 3 & 1 \\
\hline Females & 3 & 3 & 0 & 1 \\
\hline Total (\%) & $07(38.9)$ & $06(33.3)$ & $03(16.7)$ & $02(11.1)$ \\
\hline
\end{tabular}

Key: ES/IS: Epileptic/Infantile Spasms; LGS: Lennox-Gaustaut Syndrome; LKS: Landau-Kleffner Syndrome; DS: Doose Syndrome

Mean duration of treatment was $46.56( \pm 4.55)$ months. Only 5 of the cases $(27.8 \%)$ have achieved a seizure control lasting more than 6 months, following commencement of treatment.

Only half (50\%) of the cases are still on follow up. Seven (38.9\%) of the cases have been lost to follow-up, while 2 (11.1\%) of cases were transferred to adult Neurology care due to attainment of age beyond 15-years (based on the Hospital policy).

A summary of the characteristics of the cases recorded is shown in Table 1 and Table 2 below:

\section{Infantile/epileptic spasms}

Seven (7) children were diagnosed with ES/IS. Their seizure presentation (semiology) was varied, but the common identified features were history of adverse perinatal events (mostly birth asphyxia), onset of abnormal body jerks mostly described as sudden contraction of the trunk, extension and elevation of the arms (epileptic spasms), lasting few seconds and occurring in several times. All the patients had associated developmental delays. The predominant EEG findings were slow and disorganized (chaotic) background, with high amplitude slow waves and spikes that are mostly asynchronous, typically described by Gibbs as hypsarrhythmia. Multifocal spikes and sharp waves are also common [14].

\section{Lennox-Gastaut syndrome}

This was the second most common syndrome identified, characterized by multiple seizure forms/changing seizure patterns that are severe and refractory to treatment. Six (6) cases of LGS were recorded in this study. Their common seizure semiology includes tonic, GTC, myoclonic, atypical absence and a tonic forms. Significant developmental delays are common presenting features, including psychomotor delays [14]. Up to $33 \%$ of the cases in this study had prior features of ES/IS, as similarly reported by others [14].

EEG features identified include diffuse background slowing, generalized burst of spikes and wave discharges, focal and multi-focal epileptiform discharges as well as other nonspecific EEG findings.

\section{Doose syndrome (Myoclonic-astatic epilepsy)}

This syndrome is characterized by myoclonic seizures followed immediately by atonia. The atonia causes a drop attack or head nodding. Two (2) cases were recorded in this study, from same family (a brother and a sister) but presented at different times of onset. Two of the siblings have now attained a good seizure control with multiple anticonvulsant therapy, over a long period of follow up. Inter-ictal EEG features demonstrated among the cases include irregular slow wave and spike discharges and other non-specific features as reported in the literature.

\section{Landau-Kleffner syndrome (epileptic aphasia)}

This syndrome is characterized by epileptic seizures and acquired language problems. All the three (3) cases in this study had premorbid normal development, although up to $13 \%$ cases could have preexisting language problem [15]. The associated clinical seizure types varied. Hearing function was normal in all the cases and brain imaging findings were insignificant.

EEG findings were mainly non-specific epileptiform features, commonly over the parietal and temporal areas. All the 3 cases of LGS were lost to follow-up after achieving fair seizure control, though without significant speech/language improvement.

The other reported forms of childhood EE syndromes (4) were not recorded in this study, thus were not discussed in this paper. They include: Dravet syndrome (severe myoclonic epilepsy of infancy), Continuous spike waves of slow sleep (CSWSS), Ohtahara syndrome (Early infantile epileptic encephalopathy) and the later added severe epilepsy with multiple independent spike foci [15]. Thus, they are not included in this report.

\section{Discussion}

Childhood epileptic encephalopathies though not very common, are still prevalent in clinical practice. It is known 
that any form of generalized, longstanding epilepsy can potentially damage the brain $[16,17]$, but in syndromes of $E E$, the damaging effect on the brain is always present $[7,10,18]$. Thus, cognitive problems in epilepsy can be multifactorial and/or bidirectional as proposed by Helmstaedter and Witt [16]. Also, the clinical presentation of these epileptic encephalopathies is usually age-dependent and syndrome specific [18].

We recorded four (4) different types of EE syndromes in this study, mainly based on their electro-clinical presentations. Infantile/epileptic spasm was the most prevalent syndrome recorded. This is in agreement with the report by Ware, et al. [4] In Australia. Similarly, [19] have reported West syndrome/epileptic spasm to be the second most common epilepsy syndrome seen in an electrophysiology laboratory in Southeast Nigeria. All the recorded epileptic spasm cases in our study were associated with either developmental delays, developmental stagnation or developmental regression before or after the onset of clinical seizures. This finding has been documented by others $[4,20]$.

Unlike primary/idiopathic epilepsies, most epileptic encephalopathies may have an obvious underlying CNS pathology ranging from perinatal brain insults, brain cortical malformations or other imaging abnormalities. The predominant aetiologies reported also varied from region to region, with perinatal asphyxia being a common factor in developing world $[19,21]$, as also revealed by our study. Perinatal asphyxia is still very prevalent in the developing world including Nigeria and could be a frequently leading cause of symptomatic epilepsies [5] including epileptic encephalopathies as demonstrated in this study. There were no clear family histories of epilepsy in majority of the cases we recorded, with the exception of the 2 siblings with Doose syndrome, whose parent also didn't report any known family history of epilepsy.

Structural brain malformations and inborn errors of metabolism are more reported in the developed world [8]. This is perhaps due to their ability to diagnose most of the subtle brain cortical malformations as well as to carryout metabolic screen to establish such diagnoses. This ability is usually lacking in the developing countries due to lack of the appropriate facilities.

Although the underlying aetiology for childhood EE is diverse $[5,6]$, identifying a definite aetiology may be fraught with challenges in areas where sophisticated diagnostic facilities are scarce or nonexistent, as in many third world countries, including Nigeria. Thus, presumptive aetiologic diagnosis may be solely based on clinical history and physical findings. Investigations such as metabolic screen, genetic studies and functional neuro-imagings are still a mirage for most developing countries.

Even the conventional neuroimaging such as CT and MRI are mostly prohibitive due to poverty and too much of out of pocket spending on health care due to lack of social health insurance in these low-income countries. Russo, et al. [13] have therefore advised that "in the current clinical practice, first try to make the diagnosis and then start to look for the aetiology, including genetic abnormalities".
The characteristic clinical and EEG features of these EE are helpful in establishing the diagnosis. Also, they share common clusters of features, such as severe and frequent seizure attacks, developmental impairments, poor response to typical anticonvulsants and persistent interictal EEG abnormalities, which make the syndromes apparent in most cases $[10,13]$.

The brain effect in EE can be functional, structural or both, and its manifestation depends on the areas of the brain that are most affected. When both developmental impairment and frequent epileptic activity are present, the syndrome is better termed developmental and epileptic encephalopathy $[4,13,20,22]$.

In this study, the predominant neuroimaging (brain CT or MRI) findings were those of features of cerebral atrophy, with three (3) other cases showing in-active hydrocephalus, cerebral calcification and areas of infarction respectively. None of the cases had findings suggestive of a cortical brain malformation.

A definitive diagnosis of aetiologic causes was limited by lack of relevant tests such as genetic tests and functional neuroimaging modalities. Similarly, Khan and Al Baradie [5] have reported up to $40 \%$ of infantile spasms cases (the commonest form in our study) showed no clear aetiology. Conversely, Stödberg, et al. [9] demonstrated an aetiologic diagnosis in up to $65 \%$ of children presenting with epilepsy syndromes in the first 2 years of life in Sweden.

The management of refractory seizures in EE is faced with multiple challenges in our environment. For example, some of the practical issues encountered among our study cohort includes: Poor availability and cost of the different anticonvulsant medications that are required, mostly in combinations (as poly therapy), poor clinical response (as compared to other idiopathic/primary epilepsies), misconceptions about the disorders as well as poverty and ignorance on the part of the caregivers.

Due to the refractory nature of EEs to treatment, most of the patients had to be on multiple anticonvulsant medications (ranging from 2-4 different anticonvulsants) in order to achieve significant seizure control, over a long period of time. This predisposes the patients to a higher chances of epilepsy related morbidity and mortality [23], as well as poor drug compliance and higher rates of loss to follow-up as revealed by this study where more than one-third of the cases were lost to follow-up. This is similar to the report by Lagunju, et al. [21] from Southwest Nigeria, where up to three quarters of their patients with epilepsy defaulted from follow up by the end of first year.

The alternative treatment with ketogenic diet is not a readily available option in our setting, due to lack of experienced dietician to handle the preparation and its potential to affect the child's growth. Two (2) of our cases (with LGS and Doose syndrome), were considered for ketogenic dieting due to poor seizure control despite multiple antiepileptic drugs in combinations, but that was not feasible due to lack of a dietician experienced in preparing and handling ketogenic diet, in our center and nearby Hospitals. 
None of the cases was considered for a surgical treatment of the epilepsy, which is usually considered as the last resort.

Early diagnosis and intervention have been shown to improve seizure control and the psychomotor outcome even in patients with EE [15]. However, this advantage is likely to be missed in most developing world due to late presentation as demonstrated in this study, where a significant delay was observed, between the time of onset of seizures and hospital presentation of the cases.

\section{Conclusion}

This study has revealed that pediatric epileptic encephalopathies are prevalent in our society, with West syndrome and LGS leading the list of identified cases. Perinatal asphyxia was the single main risk factor identified. Late hospital presentation and late diagnosis was also observed among the cases.

\section{Recommendation}

There is need for more sensitization campaigns on improved health seeking behavior, prevention of birth asphyxia and early hospital presentation of children with early onset seizures.

\section{References}

1. Engel J Jr, ILAE (2001) A proposed diagnostic scheme for people with epileptic seizures and with epilepsy. Report of the ILAE Task force on classification and terminology. Epilepsia 42: 796-803.

2. Engel J Jr (2006) ILAE classification of epilepsy syndromes. Epilepsy Research 70: S5-S10.

3. Stafstrom CE, Kossoff EH (2016) Epileptic encephalopathy in infants and children. Epilepsy Currents 16: 273-279.

4. Ware TL, Huskins SR, Grinton BE, et al. (2019) Epidemiology and etiology of infantile developmental and epileptic encephalopathies in Tasmania. Epilepsia Open 4: 504-510.

5. Khan S, Al Baradie R (2012) Epileptic encephalopathies: An overview. Epilepsy Res Treat 2012: 403592.

6. Jain P, Sharma S, Tripathi M (2013) Diagnosis and management of epileptic encephalopathies in children. Epilepsy Research and Treatment 2013: 9.

7. Korczyn AD, Schachter SC, Brodie MJ, et al. (2013) Epilepsy, cognition and neuropsychiatry (Epilepsy, brain and mind, part 2). Epilepsy Behav 28: 283-302.

8. Hwang S-K, Kwon S (2015) Early-onset epileptic encephalopathies and the diagnostic approach to underlying causes. Korean J Pediatr 58: 407-414.
9. Stödberg T, Tomson T, Barbaro M, et al. (2020) Epilepsy syndromes, etiologies and the use of next-generation sequencing in epilepsy presenting in the first 2 years of life: A population based study. Epilepsia 61: 2486-2499.

10. Gonsales MC, Montenegro MA, Soler CV, et al. (2015) Recent developments in the genetics of childhood epileptic encephalopathies: impact in clinical practice. Arq Neuropsiquiatr 1-13.

11. Srivastava S, Sahin M (2017) Autism spectrum disorder and epileptic encephalopathy: Common causes, many questions. J Neurodev Disord 9: 23.

12. Myes CT, Mefford HC (2015) Advancing epilepsy genetics in the genomic era. Genome Medicine 7: 91.

13. Russo A, Gobbi G (2020) A reflection on the role of genetics in the concept of "epileptic encephalopathy", as emerged from the most recent ILAE classification of epilepsy. Italian Journal of Pediatrics 46: 2 .

14. Laoprasert P (2011) Atlas of Paediatric EEG. McGraw-Hill Companies 529-611.

15. Kural Z, Ozer AF (2012) Epileptic encephalopathies in adults and childhood. Epilepsy Research and Treatment 2012: 205131.

16. Helmstaedter C, Witt JA (2017) Epilepsy and cognition: A bidirectional relationship? Seizure 49: 83-89.

17. Cheng D, Yan X, Xu K, et al. (2020) The effects of interictal epileptiform discharges on cognitive and academic performance in children with idiopathic epilepsy. BMC Neurology 20: 233-239.

18. He N, Lin Z-J, Wang J, et al. (2018) Evaluating the pathogenic potential of genes with de novo variants in epileptic encephalopathies. Genetics in Medicine 21: 17-27.

19. Ogoke CC, Igwe WC, Ike II, et al. (2019) Profile of childhood epilepsies and syndromes seen in an electrophysiologic laboratory in southeast Nigeria. Int J Clin Pediatr 8: 32-36.

20. Vlaskamp DRM, Saw BJ, Burger R. et al. (2019) SYNGAP1 encephalopathy: A distinctive generalized developmental and epileptic encephalopathy. Neurology 92: e96-e107.

21. Lagunju IA, Fatunde OJ, Takon I, et al. (2009) Profile of childhood epilepsy in Nigeria. Journal of Pediatric Neurology 7: 135-140.

22. Raga S, Specchio N, Rheims S, et al. (2021) Developmental and epileptic encephalopathies: Recognition and approaches to care. Epileptic Disord 23: 40-52.

23. Dalic L, Cook MJ (2016) Managing drug-resistant epilepsy: challenges and solutions. Neuropsychiatric Disease and Treatment 12: 2605-2616.

DOI: $10.36959 / 595 / 430$

Copyright: (c) 2021 Ahmad MM, et al. This is an open-access article distributed under the terms of the Creative Commons Attribution License, which permits unrestricted use, distribution, and reproduction in any medium, provided the original author and source are credited. 